\title{
Tenacidade à Fratura Translaminar Dinâmica de Laminados Compósitos de Fibras de Carbono e Resina Epóxi de Grau Aeronáutico
}

\author{
José R. Tarpani, Maria C. A. Gatti \\ Departamento de Engenharia de Materiais, Aeronáutica e Automobilística, EESC-USP
}

\begin{abstract}
Resumo: A tenacidade à fratura translaminar dinâmica de quatro laminados compósitos de fibras de carbono e resina epóxi foi determinada nas velocidades de impacto de 2,25 e 5,52 m/s, sob as temperaturas de $-70,+25$ e $+100{ }^{\circ} \mathrm{C}$. Concluiu-se que a tenacidade à iniciação da fratura dos laminados confeccionados com fibras na forma de fita unidirecional é, em qualquer condição de ensaio, muito superior à dos laminados manufaturados com fibras dispostas na forma de tecido bi-direcional. Quanto à tenacidade à propagação de danos, constatou-se que o laminado fita processado a $180{ }^{\circ} \mathrm{C}$ é o mais indicado para operar sob impacto em temperaturas intermediárias, enquanto que o manufaturado a $120^{\circ} \mathrm{C}$ é a melhor opção para trabalhar sob ambos os extremos do intervalo de temperatura avaliado.
\end{abstract}

Palavras-chave: Impacto instrumentado Charpy, laminado compósito aeronáutico, tenacidade à fratura dinâmica.

\section{Translaminar Dynamic Fracture Toughness of Aeronautic Grade Composite Laminates Made With Carbon Fiber-Epoxy Resin}

\begin{abstract}
The translaminar dynamic fracture toughness of four carbon fiber - epoxy resin composite laminates was compared at the impact velocities of 2.25 and $5.52 \mathrm{~m} / \mathrm{s}$, under the temperatures of $-70,+25$ and $+100{ }^{\circ} \mathrm{C}$. It has been concluded that the initiation fracture toughness of unidirectional tape laminates is quite higher than bidirectional woven fabric composites, despite the testing conditions. In regard to the damage propagation toughness, it has been shown that the tape laminate processed at $180{ }^{\circ} \mathrm{C}$ is the best option at intermediate temperatures, whereas the tape composite manufactured at $120^{\circ} \mathrm{C}$ is the most suitable to operate under impact at both the extremes of the temperature range evaluated.
\end{abstract}

Keywords: Instrumented Charpy impact testing, aeronautical composite laminate, dynamic fracture toughness.

\section{Introdução}

Os tradicionais laminados compósitos confeccionados com fibras de carbono e resina epóxi (designados de ora em diante simplesmente por C-EPX), em que fibras contínuas de carbono reforçam uma matriz epoxídica, satisfazem os atuais requisitos de projetos estruturais de aeronaves subsônicas.

Entretanto, o necessário aumento de eficiência estrutural das futuras gerações de aeronaves comerciais visando a economia de combustível e o atendimento das exigências ambientais demandarão projetos mais otimizados, calcados em critérios muito mais sofisticados que os providos pela Mecânica dos Sólidos, como, por exemplo, os critérios de tenacidade da Mecânica da Fratura $^{[1]}$

Uma limitação amplamente reconhecida das estruturas laminares é a sua baixa resistência a impactos transversais ${ }^{[2]}$. Entretanto, o desempenho dos laminados compósitos aeronáuticos sob trincamento translaminar dinâmico tem sido até o momento muito pouco apreciado.

A partir dos conceitos da Mecânica da Fratura, é possível inferir o grau de segurança que um componente estrutural possui contra fraturas catastróficas em serviço. Um dos parâmetros da Mecânica da Fratura Elásto-Plástica (MFEP) empregado na estimativa da resistência dos materiais à fratura lenta (quase-estática), sob condições de plasticidade à frente da trinca, é a integral-J $\mathrm{J}^{[3]}$, que originou o critério de tenacidade à iniciação da fratura $J_{i c}$. Uma vez determinado experimentalmente, $J_{i c}$ deve ser validado de modo a garantir que a zona plástica (ou elástica não-linear) na ponta da trinca, englobando a zona de processos de fratura, seja pequena quando comparada às dimensões do componente. Caso esta condição seja satisfeita, assume-se que a tenacidade $J_{i c}$ é uma verdadeira propriedade do material, independentemente do tamanho e da geometria do componente, sendo então designada $J_{I c}$ e garantindo uma condição de deformação predominantemente plana no evento da fratura.

No presente trabalho, os conceitos da MFEP tradicional, por intermédio da integral-J, são estendidos ao regime de carregamento dinâmico sob impacto Charpy de laminados compósitos possuindo defeitos translaminares, dando origem ao critério de início da fratura rápida $J_{i d}$ É realizada uma comparação, numa base de propriedade mecânica absoluta (i.e., por unidade de volume do corpo de prova), da tenacidade à fratura $J_{i d}$ de quatro laminados convencionais C-EPX, sob duas taxas de carregamento e em diversas temperaturas. Busca-se, desta maneira, realizar um avanço significativo frente ao trabalho pioneiro de Fernandez-Cantelli et al. ${ }^{[4]}$, que avaliou a tenacidade à fratura translaminar dinâmica de laminados compósitos C-EPX no regime elástico linear, aplicando os conceitos do fator-K de intensidade de tensões elásticas lineares.

\section{Materiais e Corpos de Prova}

\section{Laminados C-EPX}

Foram ensaiados quatro tipos de laminados C-EPX supridos pela Embraer S/A já na suas formas finais, prontas e acabadas. Em dois dos laminados, as fibras originalmente pré-impregnadas com resina epóxi estavam na forma de fita unidirecional (FT), sendo que o empilhamento das lâminas individuais respeitou um arranjo $\left[0 / 90^{\circ}\right]_{7 \mathrm{~S}}$. Os outros dois laminados foram também manufaturados

Autor para correspondência: José R. Tarpani, Departamento de Engenharia de Materiais, Aeronáutica e Automobilística, Universidade de São Paulo, Av. Trabalhador São-carlense, 400, Parque Arnold Schimidt, CEP: 13566-590, São Carlos, SP, Brasil. E-mail: jrpan@sc.usp.br 
com fibras pré-impregnadas com epóxi, porém desta feita as fibras estavam dispostas na forma de tecido (TC) bi-direcional segundo o padrão de trançamento 8HS (eight harness satin), sendo que o empilhamento das lâminas obedeceu a arquitetura $\left[0 / 90^{\circ}\right]_{14}$, de maneira a que os laminados FT e TC guardassem máxima similaridade possível para fins de comparação recíproca.

Em todos os casos, a espessura final dos laminados foi nominalmente de $5 \mathrm{~mm}$.

Duas classes de resina epóxi foram avaliadas. A primeira exigiu o processamento dos laminados C-EPX à temperatura de $120{ }^{\circ} \mathrm{C}$ e pressão de $420 \mathrm{kPa}$, e a segunda demandou, respectivamente, $180{ }^{\circ} \mathrm{C}$ e $700 \mathrm{kPa}$ aplicados na manufatura dos compósitos. A resina curada a $180{ }^{\circ} \mathrm{C}$ era tenacificada com termoplástico.

Detalhes adicionais sobre os laminados C-EPX processados por bolsa de vácuo em autoclave, incluindo informações mais aprofundadas sobre as fases de reforço e respectivas matrizes, não foram fornecidos pela empresa fabricante. Entretanto, sabe-se que a fração volumétrica de fibras de reforço é da ordem de $60 \%$, e o percentual de vazios é mantido suficientemente baixo $(<1 \%)$ de modo a satisfazer os exigentes padrões de qualidade da indústria aeronáutica.

A nomenclatura adotada para os laminados compósitos C-EPX foi como segue:

- $\mathrm{FT} 120=$ Fita processada a $120^{\circ} \mathrm{C}$

- $\mathrm{FT} 180=$ Fita processada a $180^{\circ} \mathrm{C}$

- $\mathrm{TC} 120=$ Tecido processado a $120^{\circ} \mathrm{C}$

- $\mathrm{TC} 180=$ Tecido processado a $180{ }^{\circ} \mathrm{C}$

A análise dinâmico-mecânica dos laminados TC120 e TC180 $0^{[5]}$ indicou temperaturas de transição vítrea $\left(\mathrm{T}_{\mathrm{g}}\right)$ de, respectivamente, 126 e $177{ }^{\circ} \mathrm{C}$, confirmando que as temperaturas de cura dos polímeros termorrígidos empregados no presente estudo são muito próximas às respectivas $T_{\mathrm{g}}$.

Considerando que os laminados C-EPX eram perfeitamente balanceados relativamente à distribuição de fibras de carbono nas duas únicas direções principais $0^{\circ}$ e $90^{\circ}$, metade das fibras de reforço encontrava-se disposta na direção $0^{\circ}$ e a outra metade na direção $90^{\circ}$. Desta forma, a fração volumétrica de fibras dispostas na direção $0^{\circ}$ era de cerca de $30 \%$ em todos os laminados avaliados.

\section{Corpos de prova}

Os corpos de prova de laminados C-EPX foram providos pela Embraer S/A já nas suas dimensões finais. Todos os corpos de prova, destinados aos ensaios de impacto sob flexão em três pontos, possuíam dimensões nominais de $(5 \times 10 \times 55) \mathrm{mm}^{3}$, conforme mostra a Figura 1.

O entalhamento dos corpos de prova foi cuidadosamente realizado com um disco diamantado com $0,5 \mathrm{~mm}$ de espessura sob baixa rotação e refrigeração com água. O procedimento está em linha com praticamente todos os trabalhos da literatura versando sobre fratura translaminar em laminados compósitos convencionais submetidos a carregamento quase-estáticos, cíclicos e dinâmicos ${ }^{[4,6-9]}$.

O posicionamento do entalhe foi de tal modo a provocar a fratura translaminar dos laminados, conforma visto na Figura 1.

No caso dos laminados C-EPX FT, o entalhe foi orientado perpendicularmente às fibras dispostas na direção principal $0^{\circ}$, as quais formavam as camadas externas do material compósito. Para os laminados TC, o entalhe foi usinado ortogonalmente às fibras do urdume (orientadas na direção $0^{\circ}$ ). Desta forma, pode-se considerar que todos os corpos de prova Charpy C-EPX assumiram idêntica orientação, qual seja a LT, conforme nomenclatura originalmente estabelecida pela ASTM-E399 ${ }^{[10]}$.

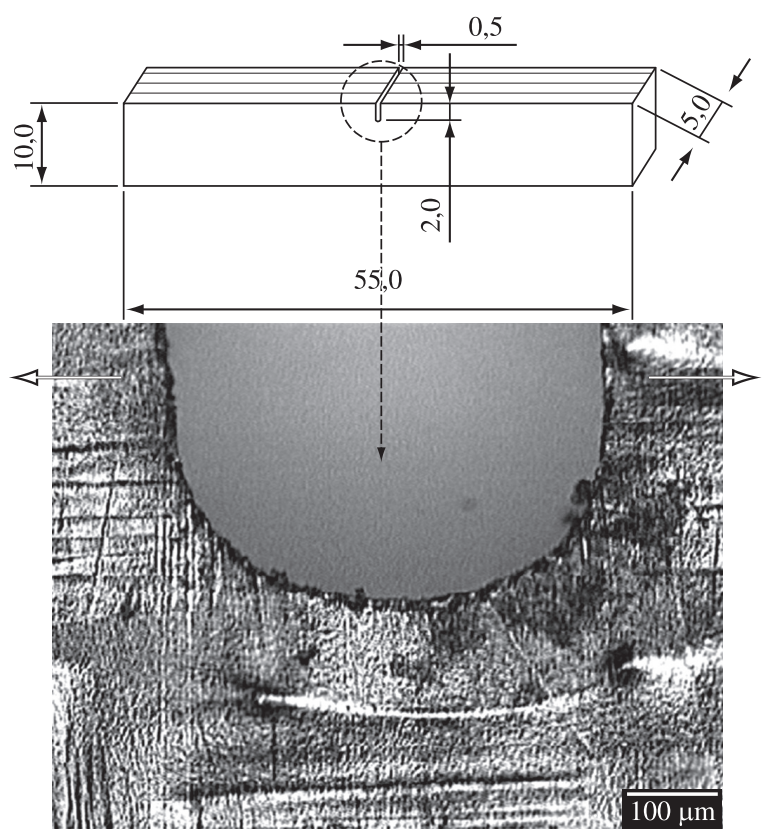

Figura 1. Geometria dos corpos de prova de impacto Charpy. No detalhe, o raio de curvatura da ponta do entalhe em um corpo de prova. Note que a fratura é translaminar e a abertura do entalhe ocorre em modo puro I de carregamento $^{[10]}$, tal como indicado pelas setas brancas.

\section{Procedimentos}

\section{Ensaio de impacto Charpy instrumentado}

Os ensaios de impacto Charpy instrumentado foram realizados em um sistema Instron-Wolpert ${ }^{\circledR}$ PW30 com fundo de escala máximo de $300 \mathrm{~J}$, integrado a um microcomputador que opera programas computacionais destinados à leitura e ao tratamento dos dados para interpretação dos resultados finais.

De acordo com Kalthoff ${ }^{[11]}$ e Morita et al. ${ }^{[12]}$, ensaios de impacto Charpy com alta energia disponibilizada pelo martelo (i.e., elevada inércia), relativamente à capacidade de absorção de energia pelo corpo de prova, garantem uma taxa de carregamento praticamente constante (sem transientes) durante todo o processo de deformação e fratura do material avaliado. Isto elimina a dependência dos resultados obtidos quanto à energia disponível no momento do impacto, embora ainda permaneça a dependência quanto à velocidade do choque mecânico.

O sistema de impacto empregado permite o registro da carga aplicada $(P)$ como função do tempo $(t)$, ou da deflexão na linha de carga $(s)$ do corpo de prova. Esta última variável é mensurada por intermédio do monitoramento do movimento do pêndulo de impacto, empregando-se na tarefa um transdutor óptico de raios infravermelhos já instalado de fábrica no sistema Instron. As oscilações originais dos sinais de carga são filtradas para sua eliminação, ou minimização, por intermédio de uma rotina Matlab ${ }^{\circledR}$, via método da Média Móvel ${ }^{[13]}$. O sistema Instron-Wolpert ${ }^{\circledR}$ PW30 realiza a aquisição de dados de carga numa frequência de $1 \mathrm{MHz}$, o que, segundo Kalthoff ${ }^{[11]}$, é mais do que suficiente para registrar a real função de carga mecânica que o corpo de prova experimenta, independentemente do material estrutural de engenharia avaliado (incluindo aí os materiais compósitos), com as oscilações naturais de carga inerentes ao processo.

Os ensaios de impacto foram conduzidos de acordo com diretrizes da norma ASTM-E23 ${ }^{[14]}$, que estabelece um vão de $40 \mathrm{~mm}$ entre os dois pontos de apoio do corpo de prova. A opção 
por esta norma dedicada ao ensaio de impacto pendular de materiais metálicos se deu pelo fato de permitir a confecção de corpos de prova de relativamente pequenas dimensões, possibilitando a confecção de um maior número de corpos de prova e, portanto, maior confiabilidade de resultados.

Merece ser ressaltado que impactos sob uma velocidade de até $5,5 \mathrm{~m} / \mathrm{s}$ são eventos relativamente comuns em aeronaves em condições de serviço, ou mesmo durante sua manutenção, garantindo assim aplicabilidade dos resultados deste estudo num contexto operacional aeronáutico ${ }^{[15,16]}$.

Os laminados C-EPX foram ensaiados nas temperaturas de, respectivamente, $-70,+25 \mathrm{e}+100^{\circ} \mathrm{C}$ sob a taxa de carregamento de $2,25 \mathrm{~m} / \mathrm{s}$, e somente à temperatura ambiente no caso da velocidade de impacto de $5,52 \mathrm{~m} / \mathrm{s}$.

Conforme dados providos no item anterior, Materiais e Corpos de Prova, pode-se concluir que, sem exceções, os laminados C-EPX foram ensaiados em temperaturas bem abaixo das respectivas transições vítreas, ou seja, no regime de fratura frágil das resinas epóxi reforçadas com fibras de carbono, especialmente no caso daquela tenacificada com termoplástico.

\section{Determinação do Ponto de Iniciação do Trincamento}

Kobayashi et al. ${ }^{[17]}$ desenvolveram o método da taxa de variação da flexibilidade elástica de um corpo de prova impactado sob flexão em três pontos para determinar o ponto de início de crescimento do defeito pré-existente. A expressão básica do método é representada pela Equação 1:

$$
\frac{\Delta C}{C}=\frac{C-C_{e l}}{C_{e l}}
$$

onde $\Delta C / C$ é taxa de mudança da flexibilidade elástica, $C$ é a secante da flexibilidade e $C_{e l}$ é a flexibilidade elástica.

Essencialmente, o método consiste em se registrar a carga $(P)$ e o deslocamento $(s)$ aplicados ao corpo de prova e determinar o ponto em que ocorre uma súbita alteração da variável $\Delta C / C$, a qual indicaria, nos laminados compósitos, o exato momento em que se inicia a propagação de danos a partir da ponta da descontinuidade artificialmente criada no material (entalhe), conforme esquemático da Figura 2.

\section{Cálculo da tenacidade à fratura dinâmica na iniciação do trincamento, $J_{i d}$}

$\mathrm{Na}$ determinação da tenacidade à fratura sob carregamento dinâmico, $J_{i d}$, a maioria dos pesquisadores ${ }^{[18]}$ utiliza a expressão originalmente elaborada por Rice ${ }^{[3]}$ para um regime de carregamento

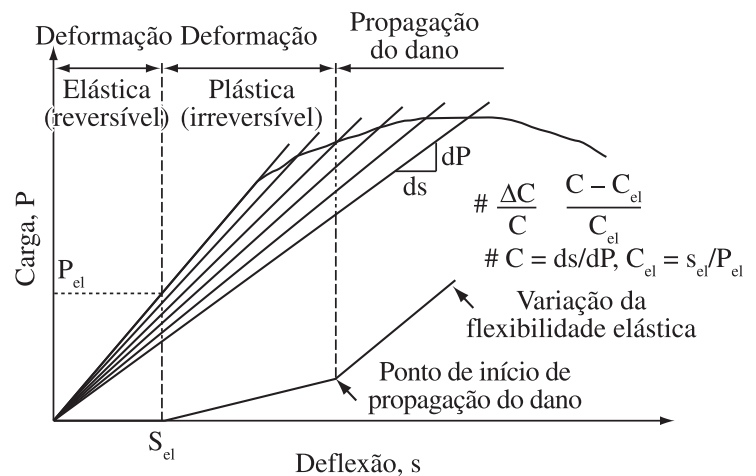

Figura 2. Método da taxa de variação da flexibilidade elástica do corpo de prova para determinação do ponto real de início de fratura (adaptado de Kobayashi et al. $\left.{ }^{[17]}\right)$. lento, ou quase-estático, empregando-se como referência o ponto de iniciação do trincamento determinado segundo o procedimento gráfico ilustrado na Figura 2. A expressão de $J_{i d}$, proposta pelos pesquisadores e posteriormente corrigida para trincas $\operatorname{rasas}^{[19]}$, é dada pela Equação 2:

$$
J_{i d}=\frac{1,46 E_{i}}{B \cdot b}
$$

onde $E_{i}$ é a energia absorvida pelo corpo de prova até o início de seu trincamento, $B$ e $b$ são, respectivamente, a espessura e o ligamento original do corpo de prova (i.e., a seção transversal íntegra do corpo de prova, à frente do entalhe).

\section{Cálculo da tenacidade à fratura dinâmica na carga máxima, $J_{m d}$}

Zhang e Shi ${ }^{[18]}$ propuseram uma simplificação do cálculo da tenacidade à fratura dinâmica dos materiais, assumindo que o defeito (trinca ou entalhe) inicia seu crescimento na carga máxima suportada pelo corpo de prova. Isto deu origem ao critério $J_{m d}$, determinado a partir da Equação 2, porém utilizando-se a energia de carga máxima $\left(E_{m}\right)$.

\section{Validação dos valores de tenacidade}

Para se determinar a validade, ou não, dos resultados de tenacidade à fratura elasto-plástica, de forma a que esta quantidade possa, ou não, ser considerada uma propriedade intrínseca e verdadeira do material, empregam-se os chamados critérios de validade (no presente caso, fornecidos na unidade de milímetros) já bem estabelecidos na literatura ${ }^{[20]}$, tendo-se como base as dimensões do corpo de prova. De modo resumido, são estes (Equação 3):

$$
B, b, W, a>25\left(J_{i d(m d)} / \sigma_{e d}\right)
$$

onde $W$ é a largura do corpo de prova, $a$ o comprimento do entalhe, e $\sigma_{e d}$ é o limite de escoamento dinâmico do material ${ }^{[21]}$ e $P_{e d}$ a carga de escoamento correspondente, onde (Equação 4):

$$
\sigma_{e d}=2,85 P_{e d} W /\left(B . b^{2}\right)
$$

Vale observar que, a despeito da fragilidade intrínseca dos laminados C-EPX, a curva de carregamento em flexão dinâmica sob três pontos de corpos de prova entalhados translaminarmente e confeccionados com estes materiais compósitos tende a apresentar um comportamento não-linear a partir de certo ponto antes da carga máxima ser atingida, particularmente em temperaturas mais elevadas, em especial no caso dos laminados FT, tal como mostra a Figura 3. Esta não-linearidade está associada basicamente a mecanismos de deformação irreversível sob esforços atuantes de tração e de compressão, e à geração e difusão de danos no material a partir da ponta do entalhe, tais como trincas na matriz, descolamento

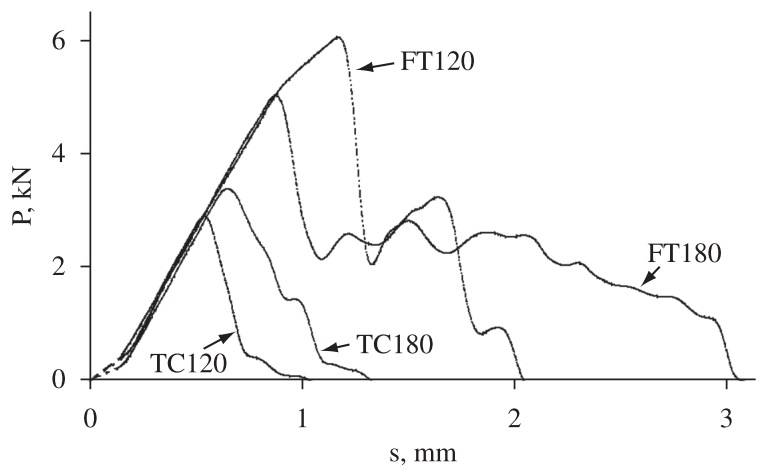

Figura 3. Curvas $P-s$ de carregamento sob impacto translaminar em flexão três pontos de laminados C-EPX a $2,25 \mathrm{~m} / \mathrm{s} \mathrm{e}-70{ }^{\circ} \mathrm{C}$. 
fibra/matriz, fratura de fibras por tração e por flambagem, e delaminações ${ }^{[22,23]}$.

Desta forma, o parâmetro $\sigma_{e d}$ foi determinado com base em uma saída da linearidade inicial da curva polida de carga $(P)$ contra deflexão do corpo de prova $(s)$, da ordem de $5 \%$, ou então, na ausência de um comportamento não-linear significativo, aquele parâmetro de escoamento (não-linearidade) foi definido na carga máxima suportada pelo corpo de prova.

Um programa computacional, contendo as formulações providas nas Equações 1-4, foi especificamente elaborado utilizando-se ferramentas Matlab $^{\circledR}$, possibilitando o polimento das curvas $P-S$ originais e a imediata geração e validação (ou não) dos resultados de tenacidade segundo as duas abordagens -J em questão, a saber, de iniciação de trincamento, $J_{i d}$, e de carga máxima, $J_{m d^{*}}$.

\section{Análise estatística}

A análise estatística realizada neste trabalho teve como referência o método $t$-Student, visto que o número de amostragem foi pequeno $(\mathrm{n}<<30)^{[24]}$.

$\mathrm{O}$ procedimento adotado envolveu a análise de uma amostra de composição conhecida, e comparação do valor t obtido com o tabelado, de modo a verificar se o resultado encontrado diferia do valor verdadeiro com uma magnitude maior do que aquela que poderia ser atribuída somente a erros casuais.

O limite de confiança empregado no estudo foi de $95 \%$, significância de 5\% com 4 graus de liberdade. De acordo com Spiegel ${ }^{[25]}$ e Achcar e Rodrigues ${ }^{[26]}$, o valor tabelado de $t$-Student é de 2,13 para 4 graus de liberdade e $95 \%$ de limite de confiabilidade. As análises realizadas foram bilaterais, empregando-se como dados de entrada os valores de tenacidade $\mathrm{J}$ e o critério de validade, os quais foram calculados de forma convencional em termos de valores médios e correspondentes desvios-padrão, como funções, respectivamente, da temperatura e da taxa de carregamento aplicada no ensaio de impacto Charpy.

A análise estatística foi realizada em comparação com duas médias para cada critério de tenacidade $\mathrm{J}$ e sua validação com base nas dimensões dos corpos de prova. Numa primeira abordagem, utilizou-se como parâmetro a variação da temperatura nos ensaios realizados sob duas distintas velocidades de carregamento. $\mathrm{Na}$ segunda abordagem, adotou-se como parâmetro a variação da velocidade de impacto para a única temperatura em que se realizaram os ensaios a $5,52 \mathrm{~m} / \mathrm{s}$, qual seja, a ambiente.

\section{Resultados e Discussão}

A Figura 4 apresenta os resultados de ensaios de tenacidade $J_{i d}$ e $J_{m d^{\prime}}$ considerando-se as várias temperaturas e as duas taxas de carregamento empregadas no estudo, de respectivamente 2,25 e $5,52 \mathrm{~m} / \mathrm{s}$. Os valores médios dos parâmetros J obtidos a partir de três ensaios para cada condição experimental são plotados, com os respectivos desvios-padrão fornecidos na forma de barras verticais.

Ensaios na velocidade de impacto de $5,52 \mathrm{~m} / \mathrm{s}$ foram realizados unicamente na temperatura ambiente, e os correspondentes resultados são indicados por círculos ou elipses tracejadas nos gráficos fornecidos de ora em diante. Note que os valores estão deslocados para a direita da temperatura ambiente, de forma a não serem confundidos com os resultados obtidos nesta mesma temperatura, porém sob uma taxa de carregamento de $2,25 \mathrm{~m} / \mathrm{s}$. A intenção do pequeno deslocamento horizontal dos dados obtidos a $5,52 \mathrm{~m} / \mathrm{s}$ foi exatamente a de possibilitar a clara comparação com os resultados determinados a $2,25 \mathrm{~m} / \mathrm{s}$.

A união dos pontos de dados levantados sob a velocidade de impacto de 2,25 m/s, por intermédio de linhas tracejadas, visa apenas indicar a tendência de comportamento dos resultados experimentais

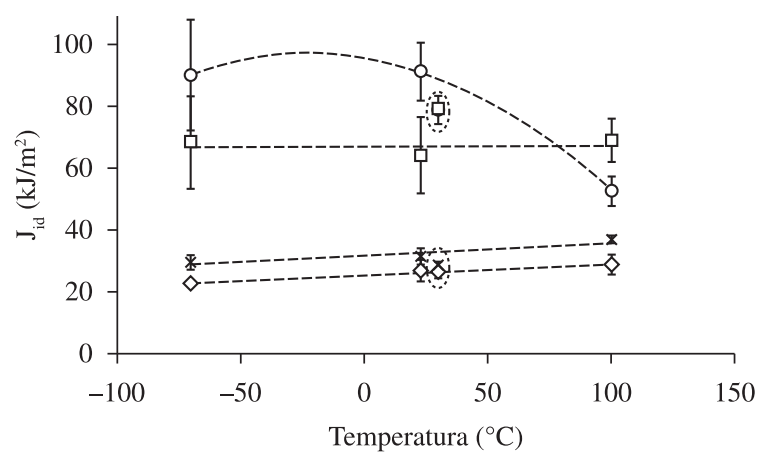

(a)

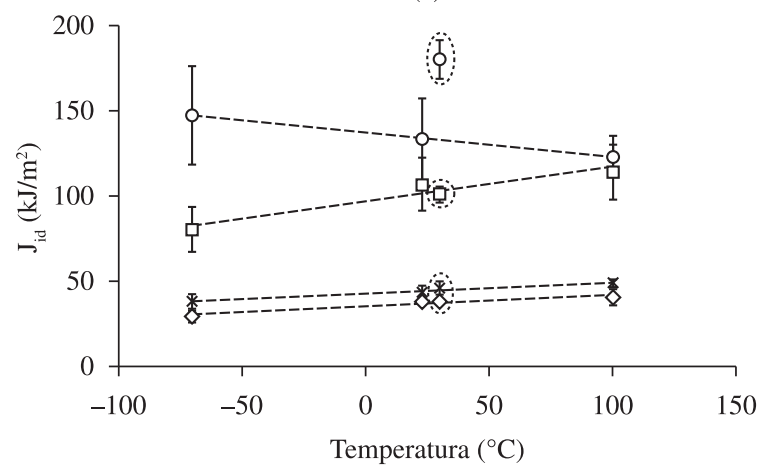

(b)

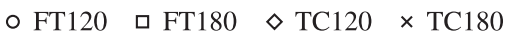

Figura 4. Resultados de tenacidade $J_{i d}$ e $J_{m d}$, para as diversas temperaturas de ensaio e velocidades de impacto: a) $J_{i d}$ a, respectivamente, 2,25 e $5,52 \mathrm{~m} / \mathrm{s}$ (na velocidade de impacto de $5,52 \mathrm{~m} / \mathrm{s}$, os pontos de dados estão envoltos por um círculo ou uma elipse tracejados); e b) $J_{m d}$ a, respectivamente, $2,25 \mathrm{~m} / \mathrm{s}$ e $J_{m d}$ a $5,52 \mathrm{~m} / \mathrm{s}$ (na velocidade de impacto de $5,52 \mathrm{~m} / \mathrm{s}$, os pontos de dados estão envoltos por um círculo ou uma elipse tracejados).

obtidos, não devendo, em princípio, ser empregada para fins de interpolação nem de extrapolação de dados.

A análise destes gráficos permite as seguintes observações, as quais são divididas na sequência em quatro seções específicas, de maneira a facilitar a tarefa do leitor.

\section{Comportamento geral}

Os laminados FT apresentam valores de tenacidade à fratura $J_{i d}$ muito superiores aos dos laminados TC. A tenacidade superior dos FT se deve provavelmente ao maior número de interfaces passíveis de delaminação (27 para os FT, contra apenas 13 interfaces dos TC), visto que a delaminação (i.e., fratura interlaminar) constitui um poderoso mecanismo de tenacificação nas estruturas laminadas. Esta possibilidade é corroborada pela Figura 5, na qual se verifica o enorme potencial de delaminação da configuração C-EPX FT, contrariamente ao laminado C-EPX TC concorrente, que não exibe qualquer tendência para a separação entre suas lâminas individuais. A hipótese levantada para os laminados C-EPX FT implica necessariamente na iniciação simultânea das fraturas transe interlaminares neste tipo de material compósito, de sorte que, a rigor, $J_{i d}$ seria um critério de iniciação de múltiplos trincamentos para esta classe de materiais estruturais.

O laminado FT120 apresenta valores de $J_{m d}$ superiores aos dos outros laminados, em especial dos tecidos. De fato, o parâmetro $J_{m d}$ já computa, no caso de materiais mais tenazes, uma boa parcela de energia associada ao estágio de difusão de danos no material, e os resultados obtidos indicam que os laminados C-EPX FT já incorporam certa parcela de crescimento de danos anteriormente à 


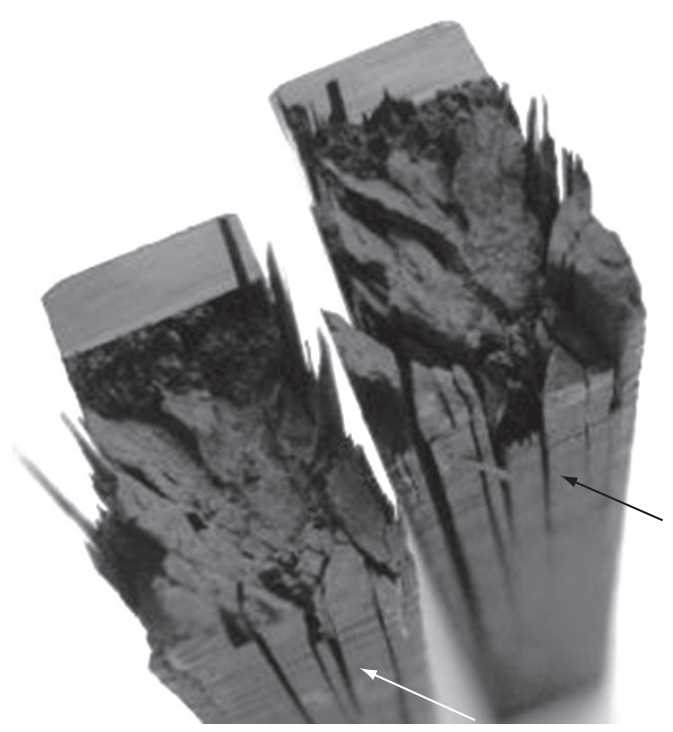

(a)

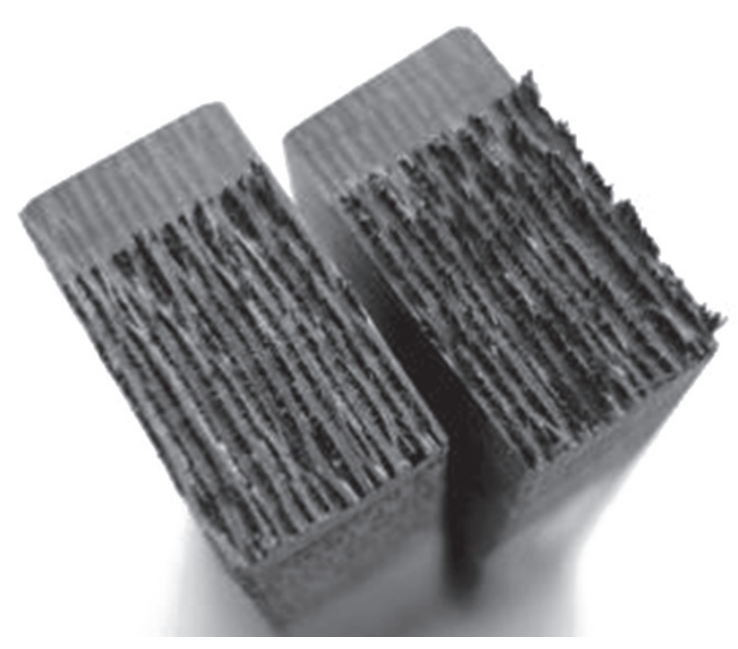

(b)

Figura 5. Aspecto de fratura dos corpos de prova: a) C-EPX FT120; e b) C-EPX TC120, ensaiados a $2,25 \mathrm{~m} / \mathrm{s}$ e $-70{ }^{\circ} \mathrm{C}$. Delaminações são indicadas por setas para a configuração C-EPX FT.

carga máxima. Esta condição pode ser matematicamente expressa por uma razão $J_{m d} / J_{i d} \gg 1$, conforme pode ser observado na Figura 6. Conclui-se desta figura que o laminado FT120 é, para fins de tenacidade à propagação de danos, a melhor escolha sob temperaturas extremas, enquanto que o FT180, é, neste sentido, a melhor opção em temperaturas intermediárias de ensaio.

Os aspectos da fratura não-frágil do C-EPX FT são claramente observados na Figura 5a, especialmente se confrontados à grande fragilidade exibida pelo laminado C-EPX TC, que é patente na Figura 5 b e que corrobora os baixos valores de $J_{i d}$ e $J_{m d}$ exibidos por esta última classe de laminado compósito. A fragilidade intrínseca dos laminados C-EPX TC, em especial daquele curado a $120{ }^{\circ} \mathrm{C}$, pode, por sua vez, ser matematicamente representada por uma razão $J_{m d} / J_{i d} \approx 1$, tal como pode ser confirmado pela análise da Figura 6. $\mathrm{O}$ comportamento de material monolítico exibido pelos laminados TC durante o processo de fratura dinâmica pode ser explicado em termos do relativamente pequeno número de lâminas compósitas individuais empregadas na sua fabricação (i.e., pequeno número de interfaces para delaminação), associado à forte interação (adesão)

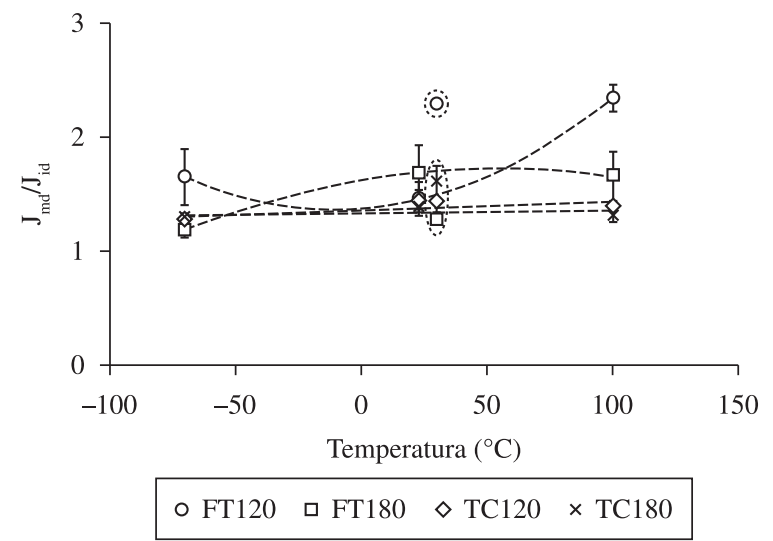

Figura 6. Razão $J_{m d} / J_{i d}$ calculada para os laminados avaliados em diversas temperaturas sob velocidade de impacto de, respectivamente, 2,25 e $5,52 \mathrm{~m} / \mathrm{s}$ (na velocidade de impacto de $5,52 \mathrm{~m} / \mathrm{s}$, os pontos de dados estão envoltos por um círculo ou uma elipse tracejados).

entre estas lâminas, tal como proporcionada pela natureza polar e termorrígida das resinas epoxídicas.

Os resultados da Figura 4 mostram que a natureza da matriz epoxídica afeta as tenacidades $J_{i d}$ e $J_{m d}$ dos laminados C-EPX, e esta influência parece depender do arranjo espacial das fibras de reforço. Os laminados FT são altamente sensíveis ao tipo de resina empregada, enquanto que os TC dependem muito pouco da mesma. Paiva et al. $^{[5]}$ realizaram ensaios de resistência à flexão, ao cisalhamento interlaminar e à compressão de laminados TC idênticos aos estudados no presente trabalho e, do mesmo modo como ora verificado para as tenacidades $J_{i d}$ e $J_{m d}$ desta arquitetura de fibras, eles também confirmaram a superioridade do desempenho do laminado TC impregnado com resina tenacificada, curada a $180{ }^{\circ} \mathrm{C}$, sobre o mesmo arranjo de fibras, porém embebido em resina epóxi padrão, curada a $120^{\circ} \mathrm{C}$. Os pesquisadores atribuíram este comportamento à melhor interface fibra/matriz alcançada pela resina tenacificada com termoplástico. Interessante observar que, no presente caso, esta tendência não foi seguida pelos laminados de fitas unidirecionais FT, visto que, quase que invariavelmente, o desempenho da resina padrão supera a da resina tenacificada. Este fato pode estar associado novamente à grande tendência à delaminação exibida por esta configuração de fibras, que seria o mecanismo controlador da tenacidade à fratura do laminado compósito. Nestas circunstâncias, o efeito alegado da melhor interface fibra/matriz da resina curada a $180{ }^{\circ} \mathrm{C}$ não é tão relevante, visto que a fratura interlaminar é basicamente controlada pela resistência mecânica da matriz polimérica termorrígida.

\section{Efeito da temperatura de ensaio}

Para uma velocidade de impacto de 2,25 m/s, a Figura 4 mostra que um aumento na temperatura de ensaio causa um significativo decréscimo em ambas as tenacidades $J_{i d}$ e $J_{m d}$ do laminado C-EPX FT120, indicando um enfraquecimento do mecanismo de tenacificação deste material durante seu aquecimento. A Figura 5a fornece indícios de que, no caso do laminado compósito C-EPX, o mecanismo de tenacificação afetado é exatamente o de delaminação. Em baixas temperaturas $\left(-70{ }^{\circ} \mathrm{C}\right.$ no caso da Figura 5a) pode-se admitir que tensões residuais substanciais surjam entre lâminas ou camadas justapostas do arranjo fita unidirecional (FT), cujas fibras estão dispostas ortogonalmente entre si. Estas tensões interlaminares resultam basicamente de variações dimensionais diferenciais induzidas termicamente, podendo favorecer a delaminação caso provoquem tração na direção da espessura do laminado. Este favorecimento da delaminação, causando em última instância à 
tenacificação do material, seria suprimido na medida em que a temperatura é incrementada, reduzindo assim os valores de $J_{i d}$ e $J_{m d^{*}}$.

Por outro lado, a presença da resina tenacificada no laminado FT180, assim como a existência de um menor número de interfaces nos laminados C-EPX TC, provavelmente inibam naturalmente o desenvolvimento de delaminações nestes laminados compósitos, de sorte que sua tenacidade não deve ser controlada por este mecanismo, e, portanto, $J_{i d}$ e $J_{m d}$ devem incrementar na medida em que estes materiais são aquecidos, conforme mostram as Figuras 4a e c.

De acordo com a análise estatística realizada, conclui-se que a tenacidade $J_{m d}$ dos laminados C-EPX é o critério de fratura provavelmente mais afetado pela variação da temperatura sob as duas taxas de carregamento empregadas.

\section{Efeito da taxa de carregamento}

Verifica-se também que o efeito da resina epóxi nos laminados C-EPX é bem mais significativo para os FT do que para os TC. Neste sentido, a resina não-tenacificada, curada a $120{ }^{\circ} \mathrm{C}$, afeta negativamente o desempenho dos FT quando do incremento da velocidade de impacto de 2,25 a 5,52 m/s à temperatura ambiente, enquanto que a matriz epoxídica tenacificada com termoplástico, curada a $180{ }^{\circ} \mathrm{C}$, é mais benéfica ao desempenho desta mesma arquitetura de fibras. Por outro lado, os laminados compósitos C-EPX TC são praticamente insensíveis à variação na taxa de deformação imposta no ensaio de impacto.

Com relação ao critério de carga máxima $J_{m d}$ dos compósitos C-EPX, à exceção do FT120, todos os laminados são insensíveis à taxa de carregamento à temperatura ambiente. De fato, o laminado FT120 apresenta uma significativa tenacificação devida ao aumento na velocidade de impacto. Analogamente, poder-se-ia agora postular que a tenacificação sob mais elevadas taxas de carregamento também seja fruto da facilitação da ocorrência de delaminação sob as condições mais severas de impacto.

Estatisticamente, verificou-se que a tenacidade $J_{i d}$ dos laminados C-EPX tende a ser o critério de fratura mais dependente da taxa de carregamento à temperatura ambiente, enquanto que a tenacidade $J_{m d}$ pouco depende daquela variável do ensaio de impacto Charpy.

\section{Validação dos resultados de tenacidade}

A Figura 7 mostra os resultados gráficos do processo de verificação da validação dos valores de tenacidade $J_{i d}$ e $J_{m d}$ por intermédio da aplicação da Equação 3, que corresponde

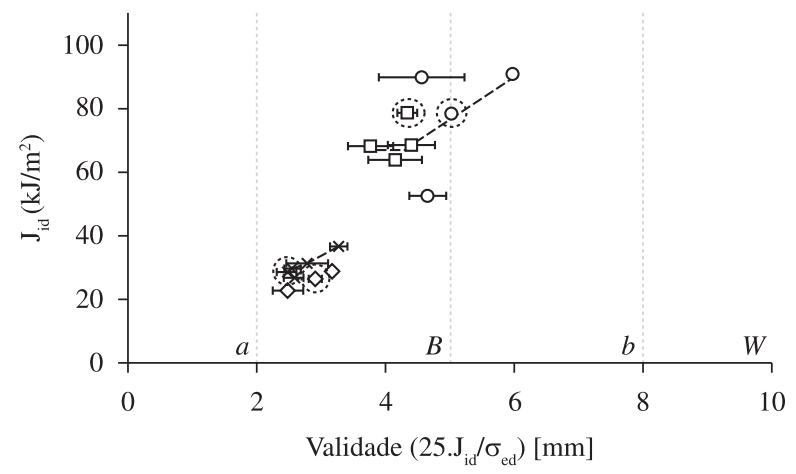

(a) matematicamente a uma inequação, visto definir uma desigualdade. O eixo das abscissas representa os valores numéricos obtidos por intermédio do termo situado do lado direito da Equação 3. Portanto, o procedimento consiste na comparação destes valores calculados com base nos resultados experimentais de, respectivamente, $J_{i d}$ e $J_{m d}$ com as dimensões significativas dos corpos de prova ensaiados sob impacto, a saber: espessura $(B=5 \mathrm{~mm})$, ligamento ( $b=W-a=8 \mathrm{~mm})$, largura $(W=10 \mathrm{~mm})$, e comprimento do entalhe $(a=2 \mathrm{~mm})$, que são grafadas na Figura $7 \mathrm{a}$.

Desta forma, caso os pontos de dados assim calculados se encontrem à esquerda do respectivo valor do critério de validação aplicado à Equação 3, seja ele $B, b, W$ ou $a$, os pontos de dados $J_{i d}$ e $J_{m d}$ estão automaticamente validados segundo aquele critério em particular, visto que a condição imposta pela Equação 3 é plenamente satisfeita. Caso contrário, ou seja, se os pontos de dados estão localizados à direita do critério de validação empregado, o requisito de dimensão mínima do corpo de prova não é satisfeito para estabelecer $J_{i d}$ e $J_{m d}$ como verdadeiras propriedades do material naquela condição de ensaio, já que uma condição minimamente admissível de predominância de deformação-plana à frente do defeito não pode ser garantida.

Os valores médios das validades J obtidas a partir de três ensaios para cada condição experimental são plotados, com os respectivos desvios-padrão fornecidos na forma de barras horizontais.

Observa-se na Figura 7 que, à exceção do comprimento do entalhe ( $a=2 \mathrm{~mm}$ ), os laminados C-EPX satisfazem, em maior ou menor extensão, praticamente todos os critérios estabelecidos em termos de dimensões mínimas necessárias do corpo de prova para o desenvolvimento de uma condição de deformação predominantemente plana à frente do entalhe. Desta forma, conclui-se que a espessura $(B)$, o ligamento $(b=W-a)$ e a largura do corpo de prova $(W)$ são suficientemente robustos para garantir $J_{i d}$ como uma verdadeira propriedade do material. Nestas circunstâncias, $J_{i d}$ passa a assumir a denominação $J_{I d}$. A rigor, dentre todos os materiais presentemente avaliados, somente o laminado FT120 tende, em algumas ocasiões, a ultrapassar os limites estabelecidos de validade da tenacidade $-\mathrm{J}$ à fratura.

Como esperado, os valores $J_{m d}$, por já embutirem em si uma boa parcela do estágio de propagação da fratura (que, a rigor, é iniciada em $J_{i d}$ ) e, consequentemente, estarem associado a uma zona de processos fratura mais ampla e difusa se comparada àquela vinculada ao critério $J_{i d}$, tendem a violar com uma certa frequência e

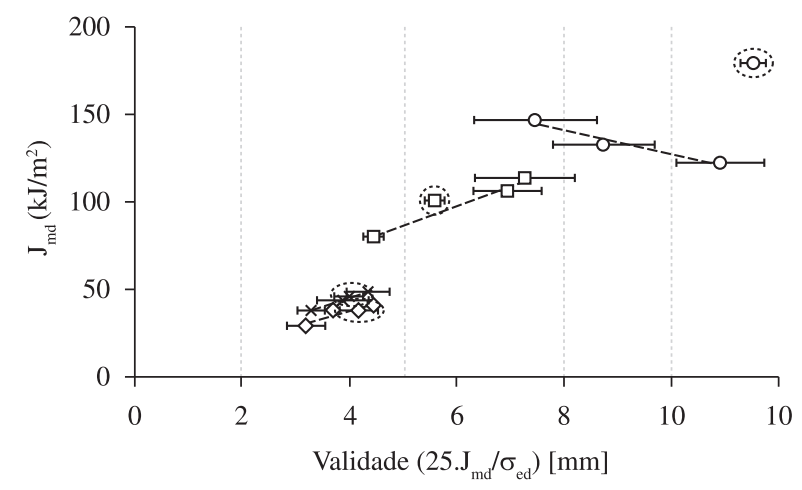

(b)

$\circ$ FT120 口 FT180 $\diamond \mathrm{TC} 120 \times \mathrm{TC} 180$

Figura 7. Critérios de validade das tenacidades $J_{i d}$ e $J_{m d}$ : a) $J_{i d}$ a, respectivamente, 2,25 e 5,52 m/s (na velocidade de impacto de 5,52 m/s, os pontos de dados estão envoltos por um círculo ou uma elipse tracejados); e b) $J_{m d}$ a, respectivamente, 2,25 e $5,52 \mathrm{~m} / \mathrm{s}$ (na velocidade de impacto de $5,52 \mathrm{~m} / \mathrm{s}$, os pontos de dados estão envoltos por um círculo ou uma elipse tracejados). Note o comportamento tipo lei de potência dos pontos de dados. 
maior extensão os demais critérios de validade impostos em termos de dimensões dos corpos de prova Charpy ensaiados.

De acordo com as análises estatísticas efetuadas neste estudo, pode-se concluir que, sob a taxa de carregamento de $2,25 \mathrm{~m} / \mathrm{s}$, o processo de validação, ou não, da tenacidade elasto-plástica (MFEP) $J_{m d}$ dos laminados C-EPX é o mais influenciado pela temperatura de ensaio.

\section{Conclusões}

A tenacidade integral-J à fratura elasto-plástica dinâmica de laminados convencionais de fibras de Carbono e resina Epóxi (C-EPX) foi determinada a partir de ensaios de impacto Charpy instrumentado. As principais conclusões do presente estudo são:

- Os laminados C-EPX FT apresentam valores de tenacidade à iniciação da fratura $J_{i d}$ de 2 a 5 vezes superiores aos dos laminados C-EPX TC;

- As elevadas razões $J_{m d} / J_{i d}$ exibidas pelos laminados C-EPX FT, oscilando entre de 1,5 e 2,5, indicam que o emprego destes laminados também se justifica pela sua elevada resistência à propagação de danos;

- As tenacidades $J_{i d}$ e $J_{m d}$ à fratura translaminar do laminado C-EPX FT são controladas pela intercedência de delaminacões, as quais podem se desenvolver já no início do processo de fratura em modo de abertura I do entalhe;

- O desempenho mecânico dos laminados C-EPX FT é sensível ao tipo de resina epóxi empregada, enquanto que o dos C-EPX TC depende muito pouco da natureza da matriz epoxídica. O uso da resina padrão favorece as propriedades de tenacidade dos laminados C-EPX FT, se comparada ao efeito exercido pela resina tenacificada;

- Salvo o critério de validação de J com base no comprimento do entalhe, os laminados C-EPX satisfazem praticamente todos os requisitos quanto ao tamanho mínimo do corpo de prova, garantindo $J_{i d}$ e $J_{m d}$ como verdadeiras propriedades dos materiais.

\section{Agradecimentos}

Às agências de fomento FIPAI e CAPES, e à empresa MIB (Materials Institute of Brazil) pelo suporte financeiro, à Embraer S/A por prover os laminados C-EPX, ao Mestre em Ciência e Engenharia de Materiais D.S. Zanetti por compartilhar seus resultados experimentais, e ao atualmente Engenheiro Aeronáutico G.A. Teti por sua assistência nos ensaios de impacto. Os professores D. Spinelli e W.W. Bose do NEMAF-EESC-USP são também reconhecidos por permitirem o uso do sistema instrumentado de ensaios de impacto Charpy.

\section{Referências Bibliográficas}

1. Wang, J. \& Karihaloo, B. L. - Compos. Struct., 32, p.453 (1995).

2. Abrate, S. - Appl. Mech. Rev., 44, p.155 (1991).

3. Rice, J. R. - ASTM STP, 536, p.231 (1973).

4. Fernandez-Cantelli, A.; Arguelles, A.; Vina, J.; Ramulu, M. \& Kobayashi, A. S. - Compos. Sci. Technol., 62, p.1315 (2002).
5. Paiva, J. M. F.; Mayer, S. \& Rezende, M. C. - Mater. Res., 8, p.91 (2005).

6. Swain, R. E.; Bakis, C. E. \& Reifsnider, K. L. - ASTM STP, 1156, p.552 (1993)

7. Underwood, J. H. \& Kortschot, M. T. - "AD-A280 101 Technical Report ARCCB-TR-94010”, US Army Armament Research, Development and Engineering Center, Watervliet, N.Y. (1994).

8. Masters, J. E. - "NASA Contractor Report 201728” (1997).

9. Castrodeza, E. M.; Ipina, J. E. P. \& Bastian, F. L. - Fatigue Fract. Eng. Mater. Struct., 25, p.999 (2002).

10. American Society for Testing and Materials. - "Standard test method for linear-elastic plain-strain fracture toughness $\mathrm{K}_{\mathrm{Ic}}$ of metallic materials, Designation ASTM-E399" (2005).

11. Kalthoff, J. F. - Compos. Part B, 35, p.657 (2004).

12. Morita, S.; Hosoi; K. \& Kobayashi, T. - "Instrumented impact testing of Polymers", in: "From Charpy to present impact testing", p.155-162, François D. \& Pineau, A. (Ed.), Elsevier Science, Oxford - UK (2002). ESIS Publication, 30.

13. Fonseca, J. S.; Toledo, G. L. \& Martins, G. A. - "Estatística aplicada", 2. ed., Editora Atlas, São Paulo, (1985).

14. American Society for Testing and Materials. - "Standard test methods for notched bar impact testing of metallic materials, Designation ASTM-E23" (2005).

15. Alves, M.; Chaves, C. \& Birch, R. S. - "Impact on aircraft", in: Anais do $17^{\text {th }}$ Brazilian Congress on Mechanical Engineering, São Paulo - SP (2003).

16. Tarpani, J. R. - "Tenacidade ao impacto Charpy de materiais de construção aeronáutica", Tese de Livre-Docência, Universidade de São Paulo, Escola de Engenharia de São Carlos, Brasil (2007).

17. Kobayahi, T.; Yamamoto, I. \& Niinomi, M. J. - Test. Eval., 21, p.145 (1993).

18. Zhang, X. P. \& Shi, Y. W. - Int. J. Fract., 81, p.195 (1996).

19. Tronskar, J. P. \& Lai, M. - Eng. Fract. Mech., 69, p.321 (2002).

20. American Society for Testing and Materials. - "Standard test method for measurement of fracture toughness, Designation ASTM-E1820" (2005).

21. Server, W. L. - J. Test. Eval., 6, p.29-34 (1978).

22. Agarwal, B. D. \& Broutman, L. J. - "Analysis and performance of fiber composites", $2^{\text {nd }}$ ed., John Wiley \& Sons, (1990).

23. Anderson, T. L. - "Fracture mechanics fundamentals and applications", $2^{\text {nd }}$ ed., CRC Press, (1995).

24. Costa Neto, P. L. O. - "Estatística", Edgar Blucher, São Paulo (1977).

25. Spiegel, M. R. - "Estatística", McGraw Hill do Brasil, São Paulo (1970).

26. Achcar, J. A. \& Rodrigues, J. - "Introdução à estatística para ciência e tecnologia”, Editora USP, São Paulo (2000).

Enviado: 08/02/10

Reenviado: 20/05/10

Aceito: $12 / 06 / 10$

DOI: $10.1590 / \mathrm{S} 0104-14282011005000004$ 